\title{
RELAÇÕES EXISTENTES ENTRE RESULTADOS SIGNIFICATIVOS DA ANALISE MATEMATICA COM FUNDAMENTOS NOS AXIOMAS DO CORPO ORDENADO COMPLETO DOS NÜMEROS REAIS
}

Evelin Maria Abreu Teixeira

Departamento de Matemática. Centro de Ciēncias Naturais e Exatas. UFSM. Santa Maria, RS.

\section{RESUMO}

Este artigo trata das relações existentes entre supremo, infimo, seqüéncia limitada monōtona, convergēncia de seqüēncia,inter valos encaixantes, teorema de Arquimedes, cobertura por abertos, subcobertura finita, ponto de acumulação, seqüēncia de Cauchy, que são resultados significativos da Anālise Matemātica com fundamento nos axiomas do corpo ordenado completo dos números reais.

SUMMARY

TEIXEIRA,E.M.A., 1980. Existing relations among significant results of the mathematical analysis based upon axioms of complete or dered body of real numbers. Ciēncia e Natura (2): 1-10.

This article treats about the existing relations among su premum, infimum, monotone and bounded sequence, convergent sequence, boxing intervals, Arquimede's theorem, conver by open, finity cover, accumulation points, Cauchy sequence and cut, significant results of the Mathematical Analysis, these results are based upon axioms of complete ordered body of real numbers.

INTRODUÇÃO

A consideração dos números reais como um corpo ordenado completo permite a obtenção de resultados significativos da Anālise Matemātica, usando apenas argumentação lōgica e axiomas do corpo or denado completo. WHITE (4) refere-se à existēncia de relações entre tais resultados. No presente trabalho são apresentadas relações en tre onze importantes propriedades da Anālise Matemática sob a forma de teoremas.

1. CORPO ORDENADO COMPLETO (4)

Existe $(\mathbb{R},+, \cdot,<)$ satisfazendo a:

i) Para quaisquer $a$ e $b$ em $\mathbb{R}, a+b=b+a$.

ii) Para quaisquer $a$, b e c em $\mathbb{R},(a+b)+c=a+(b+c)$.

iii) $H a \bar{a}$ um elemento 0 em $\mathbb{R}$ tal que $a+0=a$ para todo a em $\mathbb{R}$.

iv) Para cada a em $\mathbb{R}$ hă um elemento - a em $\mathbb{R}$ tal que $-a+a=0$.

v) Para quaisquer $a$ e $b$ em $\mathbb{R}, a \cdot b=b \cdot a$. 
vi) Para quaisquer $a$, $b$ e $c$ em $\mathbb{R}, a \cdot(b \cdot c)=(a \cdot b) \cdot c$.

vii) Há um elemento 1 em $\mathbb{R}, 1 \neq 0$, tal que para todo a em $\mathbb{R} 1 \cdot a=a$.

viii) Se actRe $a \neq 0$, há um elemento $a^{-1}$ em $\mathbb{R}$ tal que $a \cdot a^{-1}=1$.

ix) Para quaisquer $a, b$ e $c$ em $\mathbb{R}, a \cdot(b+c)=(a \cdot b)+(a \cdot c)$.

$x)$ Para todo $a \varepsilon \mathbb{R}$ há apenas três possibilidades: $a<0, a=0, a>0$.

xi) Se a e b estão em $\mathbb{R}$ e $0<a$ e $0<b$, então $0<a+b, 0<a \cdot b$.

xii) Para quaisquer $a$ e $b$ em $\mathbb{R}, a<b$ se, e somente se, $a-b<0$.

xiii) Se $A \subset \mathbb{R}$ é não vazio e majorado, então $A$ tem supremo.

2. IMPORTANTES PROPRIEDADES NO CORPO ORDENADO COMPLETO (4)

As propriedades da Anālise Matemática relativas a conjun tos, seqüencias e intervalos no corpo ordenado completo dos números reais, que serão relacionadas, são:

1. Todo conjunto, não vazio, limitado superiormente, tem supremo.

2. Todo conjunto, não vazio, limitado inferiormente, tem infimo.

3. Toda seqüência limitada, não decrescente, converge.

4. Todo seqüência limitada, não crescente, converge.

5. Para $\left[a_{1}, b_{1}\right] \jmath\left[a_{2}, b_{2}\right] \jmath \ldots \operatorname{com} \lim _{n \rightarrow \infty}\left(b_{n}-a_{n}\right)=0$ existe, e é único, $P \in \prod_{n=1}^{\infty}\left[a_{n}, b_{n}\right]$.

6. Se $x>0$ então, para qualquer $y$, existe $n \in \mathbb{N}^{*}$ tal que $n x>y$

7. Toda cobertura por aberto de um intervalo fechado $\left[c_{1}, d_{1}\right]$ qual quer tem subcobertura finita.

8. Todo conjunto, infinito e limitado, tem ponto de acumulação.

9. Toda seqüência de CAUCHY converge.

10. 0 corpo ordenado completo não pode ser coberto por dois abertos, não vazios e disjuntos.

11. Em todo corte $(A, B)$ ou $A$ tem ūitimo elemento ou B tem primeiro $\underline{e}$ lemento.

\section{DESENVOL VIMENTO}

Teorema 1. (Relaciona as propriedades 1 e 2):

Se no corpo ordenado completo todo conjunto não vazio, limitado su periormente, tem supremo então todo conjunto não vazio, limitado in feriormente, tem infimo.

Demonstração:

Se $A \subset \mathbb{R}, \mathbb{R}$ corpo ordenado completo onde todo o conjunto não vazio limitado superiormente tem supremo, $A \neq \phi$ e $A$ limitado inferiormente, então $\exists L \varepsilon \mathbb{R} \mid L \leq a$ para $\forall a \in A$. Multiplicando por $(-1): L \leq a \Rightarrow-L \geq-a$; para $\forall-a \varepsilon(-A) \exists-L \varepsilon \mathbb{R} \mid-L \geq-a$. Então $(-A)$ é limitado superiormente e, por hị 


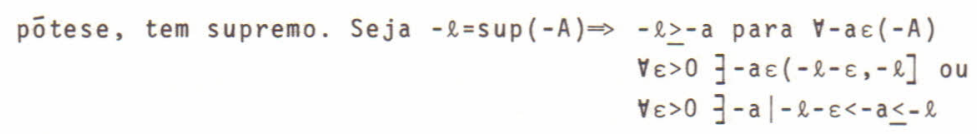

Multiplicando por $(-1)$ : $\ell \leq a$ para $\forall a \varepsilon A$

$$
\begin{aligned}
& \forall \varepsilon>0 \exists a \mid \ell \leq a<\ell+\varepsilon \text { ou } \\
& \forall \varepsilon>0] \varepsilon[l, l+\varepsilon)
\end{aligned}
$$

Logo $\ell=$ inf $A$ e A tem infimo.

Teorema 2 (Relaciona as propriedades 2 e 1 ):

Se no corpo ordenado completo todo conjunto não vazio, limitado infe riormente, tem înfimo então todo conjunto não vazio, limitado supe riormente, tem supremo.

Demonstração:

Seja $A C \mathbb{R}, \mathbb{R}$ corpo ordenado completo onde todo conjunto não vazio $1 \underline{i}$ mitado inferiormente tem infimo, $A \neq \phi$ e $A$ limitado superiormente, en tão $\exists L \varepsilon \mathbb{R} \mid L \geq a$ para $\forall a \in A$. Multiplicando por $(-1): L \geq a \Rightarrow-L \leq-a$; para $\forall-a \varepsilon(-A)]-L \varepsilon \mathbb{R} \mid-L \leq-a$. Então (-A) é limitado inferiormente e, por hí pótese, tem infimo. Seja $-\ell=\inf (-A) \Rightarrow-\ell \leq-a$ para $\forall-a \varepsilon(-A)$

$$
\begin{aligned}
& \forall \varepsilon>0]-a \varepsilon[-\ell,-\ell+\varepsilon) \text { ou } \\
& \forall \varepsilon>0]-a \mid-\ell \leq-a<\quad \ell+\varepsilon
\end{aligned}
$$

Multiplicando por $(-1): \quad l \geq a$ para $\forall a \varepsilon A$

$$
\begin{aligned}
& \forall \varepsilon>0 \exists a \mid \ell-\varepsilon<a \leq \ell \text { ou } \\
& \forall \varepsilon>0 \exists \mathrm{a} \varepsilon(\ell-\varepsilon, \ell]
\end{aligned}
$$

Logo $\ell=s u p$ A e A tem supremo.

Teorema 3 (Relaciona as propriedades 3 e 4 ):

Se no corpo ordenado completo toda seqüencia limitada, não decrescen te, converge então toda seqüência limitada, não crescente, converge.

Demonstração:

Seja $\left(a_{n}\right)$ uma seqüencia, não crescente, limitada no corpo ordenado completo onde toda seqüência limitada, não decrescente, converge. $a_{1}$ $\geq a_{2} \geq a_{3} \geq \cdots>L$. Multiplicando por $(-1):-a_{1} \leq-a_{2} \leq-a_{3} \leq \ldots<-L$. Tem-se en tão uma seqüência, não decrescente, limitada que, por hipótese, con verge. Seja $-\ell=1 \mathrm{im}\left(-a_{n}\right)$. Então $\forall \varepsilon>0 \quad \exists n_{0}\left|n \geq n_{0} \Rightarrow\right|\left(-a_{n}\right)-(-\ell) \mid \leq \varepsilon$ ou $\forall \varepsilon>0$ $\exists n_{0}\left|n \geq n_{0} \Rightarrow\right|(-1)\left(a_{n}-l\right) \mid \leq \varepsilon$ ou $\forall \varepsilon>0 \exists n_{0}\left|n \geq n_{0} \Rightarrow\right| a_{n}-l \mid \leq \varepsilon \Rightarrow \lim _{n \rightarrow \infty} a_{n}=l$. Logo $\left(a_{n}\right)$ converge.

Teorema 4 (Relaciona as propriedades 4 e 3 ):

Se no corpo ordenado completo toda seqüência limitada, não crescen te, converge então toda seqüência limitada, não decrescente, conver ge. 


\section{Demonstração:}

Seja $\left(a_{n}\right)$ uma seqüência, não decrescente, limitada no corpo ordena do completo onde toda a sequência limitada, não crescente, converge. $a_{1} \leq a_{2} \leq a_{3} \leq \ldots<L$. Multiplicando por $(-1):-a_{1} \geq-a_{2} \geq-a_{3} \geq \ldots>-L$. Tem-se então uma seqüencia, não crescente, limitada que, por hipótese, con verge. Seja $-l=1 \mathrm{im}\left(-a_{n}\right)$. Então $\forall \varepsilon>0 \exists n_{0}\left|n>n_{0} \Rightarrow\right|\left(-a_{n}\right)-(-l) \mid \leq \varepsilon$ ou $\forall \varepsilon>0$ $\exists n_{0}\left|n \geq n_{0} \Rightarrow\right|(-1)\left(a_{n}-l\right) \mid \leq \varepsilon$ ou $\forall \varepsilon>0 \exists n_{0}\left|n \geq n_{0} \Rightarrow\right| a_{n}-\ell \mid \leq \varepsilon \Rightarrow 1 \lim _{n \rightarrow \infty} a_{n}=\ell$. Logo $\left(a_{n}\right)$ converge.

Teorema 5 (Relaciona as propriedades 5,6 e 7 ):

Se no corpo ordenado completo para $\left[a_{1}, b_{1}\right] \jmath\left[a_{2}, b_{2}\right] \jmath \ldots$, com $\lim _{n \rightarrow \infty}\left(b_{n}-\right.$ $\left.a_{n}\right)=0, \exists ! P \varepsilon \prod_{n=1}^{\infty}\left[a_{n}, b_{n}\right]$ e para $\forall x>0, y$ qualquer, $\exists n \mid n . x>y$ então todaco bertura por aberto de um intervalo fechado $\left[c_{1}, d_{1}\right]$ qualquer tem sub cobertura finita.

Demonstração:

Supondo, por absurdo, que a cobertura $G:\left\{\left(a_{j}, b_{j}\right)\right.$ com $\left.i \varepsilon I\right\}$ não tenha nenhuma subcobertura finita. Tome-se o intervalo fechado $\left[c_{1}, d_{1}\right]=I_{1}$ e divida-se ao meio. Examine-se os dois intervalos $\left[c_{1}, \frac{c_{1}+d_{1}}{2}\right]$ e $\left[\frac{c_{1}+d_{1}}{2}, d_{1}\right]$ :pelo menos um desses dois intervalos não pode ser co berto por uma subcobertura finita. Bisseccione-se $I_{2}=\left[c_{2}, d_{2}\right]$ onde $I_{2}$ é um dos intervalos examinados que não tem subcobertura finita. Nova mente um dos dois intervalos $\left[c_{2}, \frac{c_{2}+d_{2}}{2}\right]$ e $\left[\frac{c_{2}+d_{2}}{2}, d_{2}\right]$ não tem subco bertura finita. Seja este $I_{3}$. Continuando o processo obtém-se uma se qüencia de intervatos $\mathrm{I}_{1}, \mathrm{I}_{2}, \mathrm{I}_{3} \ldots$ tais que cada $\mathrm{I}_{n}$ não pode ser co berto por uma subcobertura finita de G. Pela arquimedianidade $\lim _{n \rightarrow \infty}$ $\left|I_{n}\right|=0$, onde $\left|I_{n}\right|$ representa a amplitude do intervalo $I_{n}$ pois $\left|I_{n}^{n+\infty}\right|=$ $\frac{d_{1}-c_{1}}{2^{n-1}}$ e, quando $n$ cresce a partir de $n=2, \frac{d_{1}-c_{1}}{2^{n-1}}<\frac{d_{1}-c_{1}}{n-1}<\varepsilon$. Tem-se então intervalos encaixantes no corpo ordenado completo e, por hipō tese, $\exists ! P \varepsilon_{n=1}^{\infty} I_{n}$. Em particular $P_{\varepsilon} I_{1}$. Como G é cobertura de $I_{1} \exists\left(a_{i 0}\right.$, $\left.b_{i 0}\right) \varepsilon G$ que contém $P ; \log 0 a_{i 0}<P<b_{i 0}$ e como $\lim _{n \rightarrow \infty}\left|I_{n}\right|=0 \exists n_{0}$ tal que $\left|I n_{0}\right|$ $<\min \left\{P-a_{i 0}, b_{i 0^{-P}}\right\}$ conforme indicado no desenho:

\section{Ino

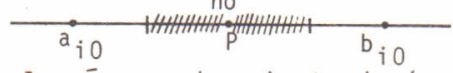

0 intervalo $I_{n 0} \bar{e}$ um subconjunto de $\left(a_{i 0}, b_{i 0}\right) \in G$ mas isto contradiz a escolha de $I_{n o}$. Assim a hipótese inicial de que não exis te subcobertura finita de $G$ que cubra $I_{1} \bar{e}$ falsa. Logo existe subco bertura finita.

Teorema 6 (Relaciona as propriedades 7 e 8 ):

Se no corpo ordenado completo toda cobertura por abertos de um inter 
valo fechado $\left[c_{1}, d_{1}\right]$ qualquer tem subcobertura finita então todo con junto, infinito e limitado, tem ponto de acumulação.

Demonstração:

Supondo por absurdo que $A C \mathbb{R}, \mathbb{R}$ corpo ordenado completo onde todaco bertura por aberto do intervalo fechado $\left[a_{1}, b_{1}\right]$ tem subcobertura fi nita, A infinito e limitado, não tenha ponto de acumulação, então $\forall$ $k \in \mathbb{R}, \exists \delta>0$ tal que $(k-\delta, k+\delta)$ contēm um nümero finito de elementos de A. Como A é limitado $\exists q_{1} \varepsilon \mathbb{R}$ e $\exists d \varepsilon \mathbb{R}$ tais que $\left[c_{1}, d_{1}\right] \jmath A$. Escolha-se convenientes $k_{j} \varepsilon\left[c_{1}, d_{1}\right]$ e $\delta_{i}$ de modo a construir uma cobertura do in tervalo $\left[c_{1}, d_{1}\right]$ e cada aberto da cobertura contēm um número finito de elementos de $A$. Tal cobertura tem subcobertura finita por hipōte se. Seja $\bigcup_{j=1}^{n}\left(k_{j}-\delta_{j}, k_{j}+\delta_{j}\right)$. Tem-se aqui a união de um nümero finito $\bar{n}$ de abertos que cobrem $A C\left[c_{1}, d_{1}\right]$ com cada aberto contendo um número $f_{i}$ nito de elementos de $A$, isto significa que $A$ tem um número finito de elementos, o que é um absurdo pois $A \bar{e}$ infinito. Logo $A$ tem ponto de a cumul ação.

Teorema 7 (Relaciona as propriedades 8 , 9 e 6 ):

Se no corpo ordenado completo todo conjunto infinito e limitado tem ponto de acumulação então toda seqüência de CAUCHY converge e para $\forall x>0, Y$ qualquer, $\exists n \mid n . x>y$.

Demonstração:

Já se sabe que toda seqüência de CAUCHY é limitada e que toda seqüen cia de CAUCHY cujos termos constituem um conjunto finito, converge. Falta então provar que toda seqüência de CAUCHY no corpo ordenado com pleto, cujos termos constituem um conjunto infinito, converge. Seja A o conjunto dos termos da seqüencia de CAUCHY. A é infinito e, por hipōtese, tem ponto de acumulação. Seja $P$ este ponto, $\log 0 \forall \varepsilon>0$ $(P-\varepsilon, P+\varepsilon)$ contēm infinitos termos $a_{n}$ da seqüência. Será mostradoque a seqüencia converge para $P$. Ora, se existem infinitos $a_{n} \varepsilon(P-\varepsilon, P+\varepsilon)$ para $\forall \varepsilon>0$ então existem finitos $a_{n} \notin(P-\varepsilon, P+\varepsilon)$ pois a seqüéncia é de CAUCHY. Seja $n_{0}$ o indice a partir do qual todos $a_{n}$ estão dentro do intervalo $\forall \varepsilon>0 \exists n_{0} \mid a_{n} \varepsilon(P-\varepsilon, P+\varepsilon)$ para $n \geq n_{0}+1$, ou seja, $\forall \varepsilon>0 \exists n_{0} \mid n>n_{0}$ $\Longrightarrow\left|a_{n}-P\right|<\varepsilon$ de modo que $\lim _{n \rightarrow \infty} a_{n}=P$ e a seqüência de CAUCHY no corpo or denado completo converge. Resta provar que para $\forall x>0, y$ qualquer,com $x$ e y no corpo ordenado completo onde todo conjunto limitado e inf $\underline{j}$ nito tem ponto de acumulação, $\exists n \mid n \cdot x>y$. Supondo, por absurdo, que $\exists x>0$, y qualquer, tal que para $\forall n \Rightarrow n . x<y$. Então $0<n . x<y$ pois $n \in \mathbb{N}$ * e assim $0<1 . x<2 . x<\ldots<n . x<\ldots<y$. 0 conjunto $\{x, 2 x, 3 x, \ldots, n . x, \ldots\}$ é limitado e infinito. Por hipōtese possui ponto de acumulação. Seja $P$ este ponto: $\forall \varepsilon>0(P-\varepsilon, P+\varepsilon)$ contēm infinitos $n . x$. Seja $\varepsilon=x: P-x<n x$ $<P+x \Longrightarrow(n+1) \cdot x>P$ e $(n-1) \cdot x<P \Longrightarrow(n-1) x<P<(n+1) . x$. Assim observa-se que 
este intervalo de centro em $P$ e raio $\varepsilon=x$ sō contēm um elemento, $n . x$, o que é um absurdo pois $P$ é ponto de acumulação. Logo nosso conjunto $\bar{e}$ ilimitado superiormente: $\sqsupset n \mid n . x>y$.

Teorema 8 (Relaciona as propriedades 9 e 5):

Se no corpo ordenado completo toda seqüéncia de CAUCHY converge en tão para $\left[a_{1}, b_{1}\right] J\left[a_{2}, b_{2}\right] \jmath \ldots \operatorname{com}_{n \rightarrow \infty} 1 i m\left(b_{n}-a_{n}\right)=0, \exists \mid P \varepsilon \bigcap_{n=1}^{\infty}\left[a_{n}, b_{n}\right]$

Demonstração:

Sejam $\left.\left[a_{1}, b_{1}\right]\right]_{2}\left[a_{2}, b_{2}\right] \jmath \ldots$ intervalos encaixantes. Observa-se que $\left|a_{m}-a_{n}\right| \leq b_{n_{0}}-a_{n_{0}}$ para $m, n \geq n_{0}$. Assim $\left|a_{m}-a_{n}\right| \leq b n-a_{n}$, para $m, n \geq n^{\prime}$. C mo $\lim _{n \rightarrow \infty}\left(b_{n}-a_{n}\right)=0$ então para $\forall \varepsilon>0 \exists n_{0}^{\prime} \mid n^{\prime} \geq n_{0}^{\prime} \Rightarrow b_{n^{\prime}}-a_{n^{\prime}} \leq \varepsilon$ ou seja $\left|a_{m}-a_{n}\right|$ $<\varepsilon$ para $m, n \geq n^{\prime} \geq n_{0}^{\prime}$. Logo $\left(a_{n}\right) \bar{e}_{\infty}$ de CAUCHY e, como tal, converge. Se ja $l=\lim _{n \rightarrow \infty} a_{n}$. Prova-se que $l \varepsilon \prod_{n=1}^{\infty}\left[a_{n}, b_{n}\right]$, is to $\bar{e}, a_{n} \leq l \leq b_{n}$ :

$10 a_{n} \leq \ell$ pois se não o fosse $\exists n_{0}=i \mid \ell<a_{i}$. Mas como a seqüencia é cres cente $\ell<a_{i}<a_{i+1}<\ldots$ e para $\varepsilon=a_{i}-l$ obtém-se 0 intervalo de centro em $\ell$ e raio $\varepsilon$ contendo não mais que $(i-1)$ termos da seqüencia, o que é um absurdo pois $\ell=\prod_{n \rightarrow \infty} a_{n}$.

$20 \underline{l} \underline{b}_{n}$ pois se não o fosse $\exists n_{0}=i \mid \ell>b_{i}$, o que não pode ocorrer jā que $a_{n}<b_{i}$ para todo $n$ e novamente haveria um intervalo de centro em $\ell$ e raio $\varepsilon_{\infty}=l-b_{i}$ contendo um nümero finito de $a_{n}$ (precisamente nenhum). Logo $l \varepsilon \bigcap_{n=1}\left[a_{n}, b_{n}\right]$.

$E$ e é ünico pois se $\exists l_{1} \neq l$ tal que $\ell_{1} \varepsilon \prod_{n=1}^{\infty}\left[a_{n}, b_{n}\right]$ então $\left[\ell, l_{1}\right] c\left[a_{n}, b_{n}\right]$ e $\lim _{n \rightarrow \infty}\left(b_{n}-a_{n}\right) \neq 0$. Logo $\ell \bar{e}$ ūnico.

Teorema 9 (Relaciona as propriedades 5, 6 e 1):

Se no corpo ordenado completo para $\left[a_{1}, b_{1}\right] \jmath\left[a_{2}, b_{2}\right] \jmath \ldots$, com $\lim _{n \rightarrow \infty}$ $\left(b_{n}-a_{n}\right)=0, \exists ! P \varepsilon \prod_{n=1}^{\infty}\left[a_{n}, b_{n}\right]$ e para $\forall x>0, y$ qualquer, $\exists n \mid n \cdot x>y$, então todo conjunto não vazio, limitado superiormente, tem supremo.

Demonstração:

Seja $A \neq \phi, A C \mathbb{R}$ e limitado superiormente. Seja L uma cota superiorde A. Como $A \neq \phi$, seja $a \in A$. Bisseccionando $[a, L]=I_{l_{l}}$ e escolhendo, como $I_{2}$, $\left[\frac{a+L}{2}, L\right]$ se contiver elementos de $A$, ou $\left[a, \frac{a+L}{2}\right]$ em caso conträrio, deter mina-se $I_{1}$ e $I_{2}$. De maneira anāloga constrōi-se sucessivamente $I_{3}$, $\mathrm{I}_{4}, \ldots$ observando-se que $\mathrm{I}_{1}, \mathrm{I}_{2}, \mathrm{I}_{3}, \mathrm{I}_{4}, \ldots$ Dessa maneina $\left|\mathrm{I}_{n}\right| \Rightarrow 0$, pois foi provado no Teorema 5 que intervalos construídos fur bisseção tem sua amplitude tendendo a zero. Sendo intervalos encaixantes tem,por hipötese, um ponto ūnico comum. Seja $P$ este ponto. Prova-se agora que $P=\sup A \cdot 10 a \leq P$ para $\forall a \varepsilon A$ pois se $\exists a^{*} \varepsilon A \mid a^{*}>P$ então seja $I_{n_{0}} 0$ pri 
meiro intervalo tal que $a{ }^{*} I_{n}$. No intervalo anterior $a^{*} E I_{n_{0}-1}$ e $a{ }^{*} E$ $\left[P, b_{n_{0}-1}\right]$, o que é um absurdo, pois o $I_{n_{0}}$ não seria o escolnido.

20 $\forall \varepsilon>0 \exists$ a $\varepsilon A \mid a \in(P-\varepsilon, P]$, ou seja, $p-\varepsilon<a \leq P$, pois para $\forall \varepsilon>0 \exists n_{0} \mid$ $P-\varepsilon<a_{n_{0}} \leq P \leq b_{n_{0}}$. E, por 10 e pela construção de $\left[a_{n_{0}}, b_{n_{0}}\right], a_{n_{0}} \leq a \leq P$.

Logo $P-\varepsilon<a_{n_{0}} \leq a \leq P \Rightarrow P-\varepsilon<a \leq P$.

Portanto A tem supremo.

Teorema 10 (Relaciona as propriedades 1 e 3 ):

Se no corpo ordenado completo todo conjunto não vazio, limitado su periormente, tem supremo, então toda seqüência limitada, não decres cente, converge.

Demonstração:

Seja $\left(a_{n}\right)$ tal que $a_{1} \leq a_{2} \leq \cdots \leq a_{n} \leq \ldots<L$ uma seqüência no corpo ordena do completo onde todo conjunto, não vazio, limitado superiormente, tem supremo. 0 conjunto dos termos de $\left(a_{n}\right) \bar{e}$ limitado superiormente e portanto tem supremo. Seja $L=\sup \left\{a_{n}\right\}$, então $\exists a_{n_{0}} \varepsilon(L-\delta, L]$ para $\forall \delta$ $>0$. Sendo não decrescente $L-\delta<a_{n_{0}}<a_{n} \leq L<L+\delta$ para $n \geq n_{0}$. Logo para $\forall \delta>0$ $\exists n_{0}\left|n \geq n_{0} \Rightarrow L-\delta<a_{n}<L+\delta \Rightarrow \forall \delta>0 \exists n_{0}\right| n \geq n_{0} \Rightarrow\left|a_{n}-L\right|<\delta$ e assim $\lim _{n \rightarrow \infty} a_{n}=L$.Por tanto toda sequêencia limitada não decrescente converge.

Teorema 11 (Relaciona as propriedades 3,5 e 6 ):

Se no corpo ordenado completo toda següência limitada não decrescen te, converge então para $\left[a_{1}, b_{1}\right] J\left[a_{2}, b_{2}\right] \jmath \ldots$, com $\lim _{n \rightarrow \infty}\left(b_{n}-a_{n}\right)=0, \exists$ ! $P E_{n=1}^{\infty}\left[a_{n}, b_{n}\right]$ e para $\forall x>0$, y qualquer, $\exists n \mid n . x>y$.

\section{Demons tração:}

Sendo $a_{1} \leq a_{2} \leq a_{3} \leq \ldots<b_{1}$ observa-se que $\left(a_{n}\right)$ é uma seqüência limitada, não decrescente, e, por hipōtese, convergente. Atém disso por conver gir é de CAUCHy e o Teorema 8 jā provou que, em um corpo ordenado com pleto onde toda seqüencia de CAUCHY, $\exists ! P \varepsilon n_{n=1}^{\infty}\left[a_{n}, b_{n}\right]$, resta provar a arquimedianidade. Supondo por absurdo que $\exists a>0$ e b qualquer tal que, para $\forall n \in \mathbb{N}^{*}, n . a<b \Rightarrow n<\frac{b}{a} \Rightarrow 1<2<\ldots<n<\ldots<\frac{b}{a}$. Esta é uma seqüencia não decrescente limitada que, por hipōtese, converge. Seja $\alpha=1 i m n$. Então para $\forall \varepsilon>0 \exists n_{0}$ tal que $|n-\alpha| \leq \varepsilon$ para $n \geq n_{0}$. Seja $\varepsilon=1$; então $\exists n_{0}$ tal que $\alpha-n<1$ para $n \geq n_{0} \Longrightarrow \alpha<n+1$ para $n \geq n_{0}=0$ que $\bar{e}$ um absurdo. Portanto para $\forall a>0$, b qualquer, J $n$ tal que $n . a<b$ e o corpo ordenado comple to onde toda seqüencia limitada, não decrescente, converge é arquí mediano.

Teorema 12 (Relaciona as propriedades 1 e 10):

Se no corpo ordenado completo todo conjunto, não vazio, limitado su periormente, tem supremo então o corpo ordenado completo não pode ser coberto por dois abertos não vazios e disjuntos. 
Demonstração:

Supondo por absurdo que o corpo ordenado completo possa ser coberto por dois abertos não vazios e disjuntos $A_{1}$ e $A_{2}$. Seja $a_{2} \varepsilon A_{2}$ e $J=\{a \varepsilon$ $\left.A_{1} \mid a<a_{2}\right\}$. J é infinito pois se não o fosse seria finitoe existiriam $n$ elementos de $A_{1}$ em $J$. Tome-se o menor destes elementos:a $a_{j}$. Ele li mita inferiormente $A_{1}$ e todo entorno dele não estaria contido em $A_{1}$, ou seja, $\left(a_{j}-\delta_{j}, a_{j}+\delta_{j}\right)$ não estaria contido em $A_{1}$ para todo $\delta_{j}$, oque é um absurdo pois $A_{1} \bar{e}$ aberto. Logo $J \bar{e}$ infinito. Além disso é tam bém limitado superiormente pelo $a_{2}$, por hipótese, tem supremo. Mos tra-se agora que $\ell=s u p J$ não está em $A_{1}$ nem $A_{2}$ :

$10 \ell \notin A_{1}$, pois se $\ell \varepsilon A_{1}$, então $\exists \delta>0$ tal que $(\ell-\delta, l+\delta) \subset A_{1}$ e. $\exists a \mid l<a<a_{2}$, pois $\ell$ não.ē o último elemento de $J$. Mas a $>\ell$ é um absurdo. Logo $\ell \notin A_{1}$. $20 \ell \notin A_{2}$, pois se $\ell \varepsilon A_{2}$ então $\ell \leq a_{2}$ e, como para $\forall \varepsilon>0 \exists a \varepsilon(\ell-\varepsilon, l]$, seja $\varepsilon=\delta_{e}$ onde $\delta_{e} \bar{e}$ tal que $\left(l-\delta_{e}, l+\delta_{e}\right) C A_{2}$. Assim $\exists a \varepsilon\left(l-\delta_{e}, l+\delta_{e}\right)$ o que é um absurdo. Logo $\ell \notin A_{2}$.

Como $l \notin A_{1}$ e $l \notin A_{2}$, $A_{1}$ e $A_{2}$ não cobrem o corpo ordenado com pleto, provando que dois abertos, não vazios e disjuntos, não podem cobrir $\mathbb{R}$.

Teorema 13 (Relaciona as propriedades 10 e 11):

Se o corpo ordenado completo não pode ser coberto por dois abertos, disjuntos e não vazios, então em todo corte $(A, B)$ ou $A$ tem último ou B tem primeiro.

Demonstração:

Supondo, por absurdo, que exista um corte $(A, B)$ onde nem $A$ tem ūiti mo elemento nem $B$ tem primeiro elemento. Como $(A, B)$ é corte, $A$ e $B$ são não vazios, $A \cup B=\mathbb{R}$ e a<b para $\forall a \in A$ e $\forall b \varepsilon B$. A é aberto pois $A$ não tem ūltimo: $\left.\forall a^{\star} \varepsilon A \exists a^{* \star} \mid a^{*}<a^{* *} \Rightarrow \forall a^{*} E A\right] \delta=a^{* *}-a^{*}$ tal que ( $a^{*}-\delta$, $\left.a^{\star}+\delta\right)=\left(2 a^{\star}-a^{\star \star}, a^{\star \star}\right) C A$, de modo que $A \bar{e}$ aberto. E B não tem primeiro: $\forall b^{*} \varepsilon B \exists b^{* *} \mid b^{* *}<b^{*} \Rightarrow \forall b^{*} \varepsilon B \exists \delta=b^{*}-b^{* *}$ tal que $\left(b^{*}-\delta, b^{*}+\delta\right)=\left(b^{* *}\right.$, $\left.2 b^{\star}-b^{\star \star}\right) C B$. Logo $B$ também é aberto.

E assim os abertos $A$ e $B$ não vazios e disjuntos, correm o corpo ordenado completo, o que é um absurdo por hipótese. Logo ou A tem último ou B tem primeiro.

Teorema 14 (Relaciona as propriedades 11 e 1):

Se no corpo ordenado completo todo corte $(A, B)$ ou $A$ tem ūitimo ele mento ou $B$ tem primeiro então todo conjunto, não vazio, limitado su periormente, tem supremo.

Demonstração:

Supondo, por absurdo, que existe um conjunto limitado superiormente, não vazio, no corpo ordenado completo, que não tenha supremo. Seja $x$ tal conjunto. Então para $\forall \ell \in \mathbb{R} \exists \varepsilon>0 \mid \exists x \varepsilon(\ell-\varepsilon, l]$, ou seja, $\ell-\varepsilon<x \leq \ell$ não é satisfeita por nenhum $x \in X$. Constrói-se um corte $(A, B)$ em $\mathbb{R}$ re 
lacionado $\operatorname{com} X$. Seja $B=\{b \mid b>x, \forall x \in X\}$. Observa-se que $B \neq \phi$ porque $X \neq \phi$ e é limitado superiormente.

Seja $A=C B(A, B) \bar{e}$ corte pois:

10 P $A$ e $B$ são não vazios, pois $A \supset X, j a \bar{a}$ que $x \in X \Rightarrow x \notin B \Rightarrow x \varepsilon C B \Rightarrow x \varepsilon A$.

20 a $a b$ para $\forall a \varepsilon A$ e $\forall b \varepsilon B$ pois $a \neq b, j a \bar{a}$ que $C B \cap B=\phi$, e $a>b \Rightarrow a>x \Rightarrow a \varepsilon B$ o que è um absurdo.

30 $A \cup B=C B \cup B=\mathbb{R}$.

Provando que nem $A$ tem ūltimo elemento nem $B$ tem primeiro elemento contraria-se a hipōtese:

10 A não tem ūitimo, pois se $a^{\star}$ fosse o último elemento de $A$ :

a) $a^{\star} \varepsilon X \Rightarrow \exists x=a^{\star} \mid a^{\star} \varepsilon\left(a^{\star}-\varepsilon, a^{\star}\right]$ para $\forall \varepsilon>0$ e $a^{\star}$ seria sup $X$.

b) $a^{*} \varepsilon A-X \Rightarrow \exists \delta>0 \mid \exists x \varepsilon\left(a^{\star}-\delta, a^{*}\right]$ de modo que ou $x<a^{*}-\delta<a^{*} \Rightarrow a^{*} \varepsilon B$, o que é um absurdo, ou $a^{\star}<x$ de modo que $a^{\star}$ não è o ūitimo.

20 B não tem primeiro, pois para $\forall b^{\star} \varepsilon B \exists \varepsilon>0 \mid \exists \times \varepsilon\left(b^{\star}-\varepsilon, b^{\star}\right]$ e assim $x<b^{*}-\varepsilon<b^{*}$ de modo que $b^{*}$ não é o primeiro.

Logo, $X$ tem supremo.

\section{CONCLUSÃO}

As propriedades da Anālise Matemática relativas a conjun tos, seqüencias e intervalos no corpo ordenado completo dos números reais, cujas relações foram demonstradas, acham-se ilustradas no se guinte diagrama:

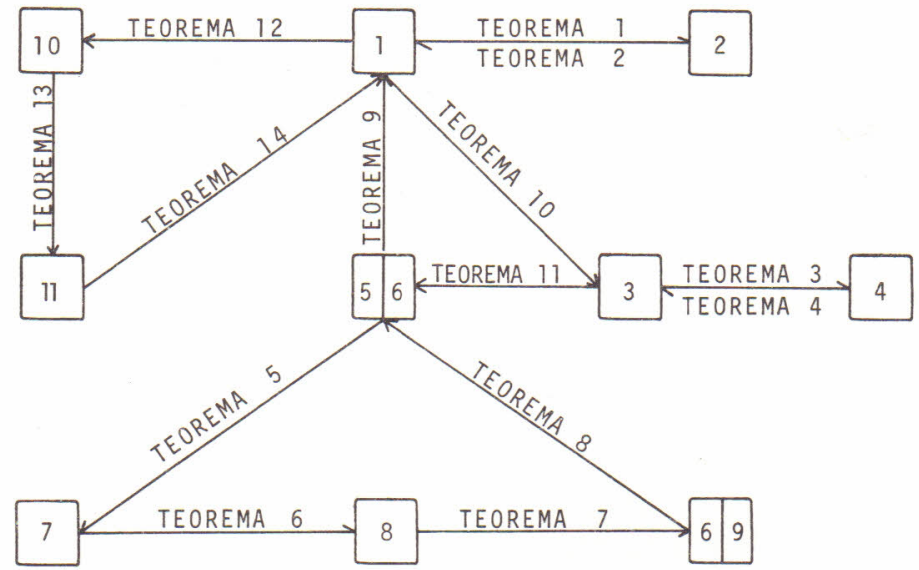

Seria possivel obter tais resultados sem depender dos axio mas do corpo ordenado completo?

E sugestão para um futuro trabalho.

Seria interessante tambēm estudar a possibilidade de obter outras relações entre as referidas propriedades e entre outras pro 
priedades da Anālise Matemātica a partir do conjunto dos números reais como um corpo ordenado completo.

\section{BIBLIOGRAFIA CITADA}

1. APOSTOL,T.M. Análisis Matematico. Barcelona. Editorial Reverte, S.A. 1976.

2. FULKS, Watson. Advanced Calculus an Introduction to Analysis. New York. John Wiley \& Sons, Inc. 1967.

3. RUDIN, Walter. Principios de Anälise Matemática. Rio de Janeiro. Ao Livro Técnico S.A. 1971.

4. WHITE,A.J. Análise Real uma Introducão, São Paulo. Editora Edgard Blücher Ltda. 1973.

Recebido em janeiro, 1980; aceito em outubro, 1980. 